\title{
Obstructive sleep apnoea is not a risk factor for incident hospitalised depression: a historical cohort study
}

\author{
Tetyana Kendzerska1,2,3,4 Andrea S. Gershon ${ }^{3,4,5}$, Gillian A. Hawker ${ }^{3,5,6}$, \\ George A. Tomlinson ${ }^{5,7}$ and Richard S. Leung ${ }^{5,8}$
}

Affiliations: ${ }^{1}$ The Ottawa Hospital Research Institute, University of Ottawa, ON, Canada. ${ }^{2}$ Institute for Clinical Evaluative Sciences, Ottawa, ON, Canada. ${ }^{3}$ Institute for Clinical Evaluative Sciences, Toronto, ON, Canada. ${ }^{4}$ Sunnybrook Research Institute, Toronto, ON, Canada. ${ }^{5}$ Dept of Medicine, University of Toronto, Toronto, ON, Canada. ${ }^{6}$ Women's College Research Institute, Women's College Hospital, Toronto, ON, Canada. ${ }^{7}$ Dept of Medicine, University Health Network/Mt Sinai Hospital, Toronto, ON, Canada. ${ }^{8}$ Dept of Medicine, St Michael's Hospital, Toronto, ON, Canada.

Correspondence: Tetyana Kendzerska, The Ottawa Hospital, Civic Campus, Room \#ASB-1-008, 1053 Carling Avenue, Ottawa, ON, K1Y 4E9, Canada. E-mail: tetyana.kendzerskalamail.utoronto.ca

@ERSpublications

Obstructive sleep apnoea has not been found to be associated with hospitalised depression http://ow.ly/sVW6309Muam

Cite this article as: Kendzerska T, Gershon AS, Hawker GA, et al. Obstructive sleep apnoea is not a risk factor for incident hospitalised depression: a historical cohort study. Eur Respir J 2017; 49: 1601361 [https://doi.org/10.1183/13993003.01361-2016].

ABSTRACT We evaluated whether obstructive sleep apnoea (OSA) was related to the incidence of hospitalisation for depression, a robust end-point that is unlikely to result from misdiagnosis.

All adults referred with suspected OSA who underwent a diagnostic sleep study at a large urban academic hospital between 1994 and 2010 and were linked to provincial health administrative data between 1991 and 2015 were included. Cox regression analysis was used to investigate the association between OSA symptoms and severity and incident hospitalised depression, the primary outcome.

Over a median follow-up of 9.7 years, 136 (1.3\%) out of 10149 participants were hospitalised for depression. A significant crude effect of OSA symptoms (waking unrefreshed and impact on memory and concentration) on hospitalised depression became nonsignificant after controlling for confounders. Apnoea-hypopnoea index was not significantly associated with the outcome: adjusted hazard ratio (33 versus 6 events $\cdot h^{-1}$ ) 1.13 (95\% CI 0.91-1.40). Factors associated with hospitalised depression were female sex, younger age, use of hypnotics, alcoholism and unemployment.

In a large clinical cohort with suspected OSA, controlling for confounders, OSA symptoms and severity were not related to the risk of hospitalisation for depression, suggesting that previously reported links between OSA and depression may be due to overlapping diagnostic criteria. However, our findings cannot exclude a potential link between OSA and milder depression. 


\section{Introduction}

An association between obstructive sleep apnoea (OSA) and depression has long been reported [1], but the answer to whether these two entities are causally related remains elusive. Both conditions are highly prevalent in the community and could be expected to coexist in a significant proportion of patients merely by chance $[1,2]$. The prevalence of depression in patients with OSA in community or sleep clinic populations has been variably estimated from $5 \%$ to $63 \%$ [3]; although some studies have reported a higher prevalence of depression among OSA patients than in the general population, others have not $[1,4]$. The high prevalence of coexisting depression and OSA might be explained by 1) shared symptomatology (e.g. daytime sleepiness, fatigue or loss of interest, poor concentration, irritability, psychomotor retardation and decreased libido) [3] or 2) common underlying risk factors (e.g. obesity, comorbid illnesses, lower socioeconomic status or smoking or alcohol use). If the association between OSA and depression is mainly due to overlapping symptoms, then these two conditions may not be linked at all, except in the sense that they are easily diagnostically confused for one another, whether by clinicians or questionnaires that probe for the same symptoms [5].

Alternatively, OSA and depression might share a multidirectional causal relationship including sleep fragmentation, biological dysregulation (inflammation, oxidative stress and neurotransmitter imbalances), metabolic syndrome, central nervous system effects of psychiatric disorders and the effect of some psychiatric medications [6].

Overlap in symptoms, risk factors and pathogenetic mechanisms may be serious obstacles in determining the presence or severity of one condition in the presence of the other. Failure to recognise and treat OSA in depressed patients may lead to inappropriate prescription of antidepressant medications and the persistence of depressive symptoms despite therapy. Similarly, failure to recognise and treat depression in OSA patients may lead to treatment failure with continuous positive airway pressure (CPAP) or other therapy. Both CPAP and mandibular advancement device treatments have been shown to be associated with small improvements in depressive symptoms as measured by validated questionnaires in individuals with OSA [7-9]. However, it remains possible that the benefit reported may not demonstrate a true effect of CPAP or mandibular advancement treatment on depression, but rather the effect of treatment on OSA symptoms falsely interpreted at baseline as depression [7].

Evidence shedding light on the longitudinal association between OSA and depression is very limited (table 1) [10-12]. The paucity of good-quality longitudinal studies of OSA and depression may be explained by a number of factors, including [13] 1) challenges in establishing a diagnosis of incident depression; 2) differences in sensitivity and specificity of the screening or diagnostic tool chosen to define depression; 3) lack of valid scales of depressive symptoms in OSA; 4) lack of a validated algorithm to define depression and OSA from administrative data; 5) differences in the prevalence of hospitalised depression and depression diagnosed by a primary care physician; and 6) potential for referral and reporting biases.

Thus, it remains unclear whether the association between OSA and depression is due to a causal relationship or due to diagnostic confusion secondary to overlapping symptoms, risk factors, diagnostic criteria or shared comorbidities. To address this knowledge gap, the current study evaluated whether OSA symptoms and severity were predictive of the incidence of hospitalisation for depression, a robust end-point that is unlikely to result from misdiagnosis in a large cohort of individuals with suspected OSA, controlling for known risk factors. We also evaluated the association between sleep-related variables and prevalent depression.

\section{Methods}

\section{Study design}

We conducted a historical cohort study using linked clinical data from a large urban academic hospital (St Michael's Hospital, Toronto, ON, Canada) collected between 1994 and 2010 and provincial health

Support statement: This project was supported by the ResMed Research Foundation. This study was supported by the Institute for Clinical Evaluative Sciences (ICES), which is funded by an annual grant from the Ontario Ministry of Health and Long-Term Care (MOHLTC). The opinions, results and conclusions reported in this paper are those of the authors and are independent from the funding sources. No endorsement by ICES or the Ontario MOHLTC is intended or should be inferred. T. Kendzerska is supported by the Canadian Respiratory Research Network (CRRN) Fellowship Training Award. Funding for training of graduate students and new investigators within the Network was supported by grants from the Canadian Institutes of Health Research (CIHR): Institute of Circulatory and Respiratory Health; Canadian Lung Association (CLA)/Canadian Thoracic Society (CTS); British Columbia Lung Association; and Industry Partners Boehringer-Ingelheim Canada Ltd, AstraZeneca Canada Inc., Novartis Canada Ltd. and GlaxoSmithKline Inc. The funding sponsors had no role in the study design, data collection and analysis, or preparation of the manuscript. Funding information for this article has been deposited with the Crossref Funder Registry.

Conflict of interest: Disclosures can be found alongside this article at erj.ersjournals.com 
TABLE 1 Characteristics of published studies that investigated a longitudinal association between obstructive sleep apnoea (OSA) and depression

Peppard et al., $2006[10]$
Wisconsin Sleep Cohort
Chen et al., 2013 [11]

Health Insurance Database, Taiwan
LANG et al., 2015 [12]

Australian Community-based cohort (abstract)

Subjects $\mathrm{n}$
Male \%
Age years
Definition of depression

Definition of OSA

Follow-up

Analyses

Effect

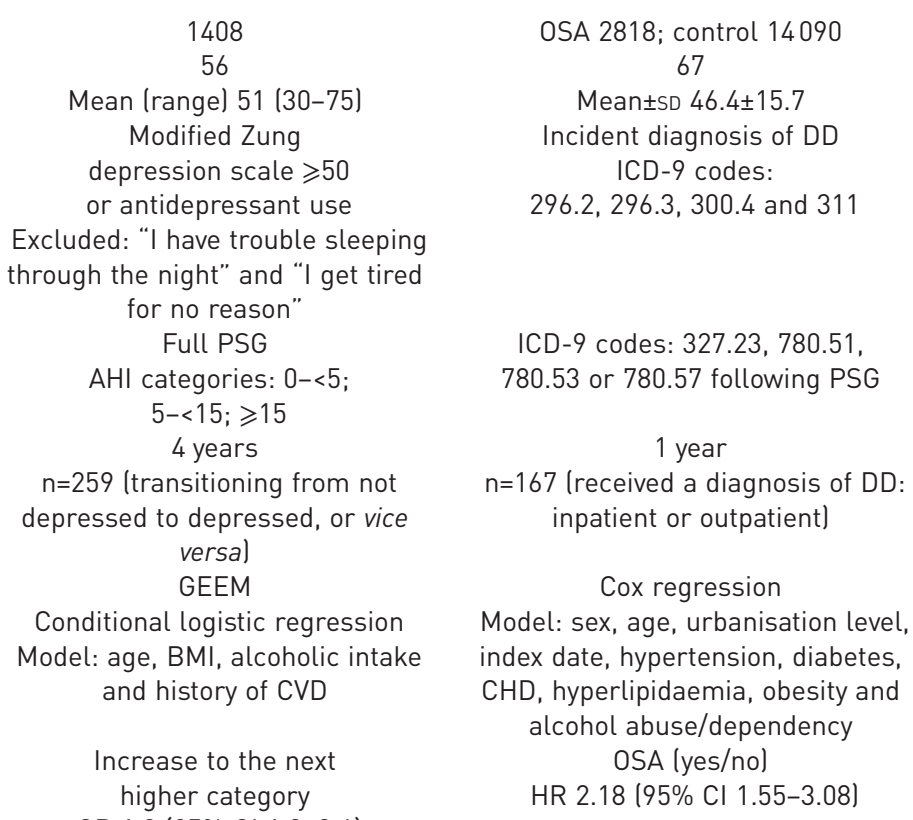

1408

56

Mean (range) 51 (30-75)

Modified Zung

depression scale $\geqslant 50$

or antidepressant use

Excluded: "I have trouble sleeping

through the night" and "I get tired

for no reason"

Full PSG

AHI categories: $0-<5$;

$5-<15 ; \geqslant 15$

4 years

$\mathrm{n}=259$ (transitioning from not

depressed to depressed, or vice versal GEEM

Conditional logistic regression

Model: age, BMI, alcoholic intake and history of CVD

Increase to the next higher category

OSA 2818; control 14090$$
67
$$

Mean \pm SD $46.4 \pm 15.7$

Incident diagnosis of DD ICD-9 codes:

296.2, 296.3, 300.4 and 311

ICD-9 codes: $327.23,780.51$, 780.53 or 780.57 following PSG

1 year

$\mathrm{n}=167$ (received a diagnosis of DD: inpatient or outpatient)

Cox regression

Model: sex, age, urbanisation level, index date, hypertension, diabetes, CHD, hyperlipidaemia, obesity and alcohol abuse/dependency OSA (yes/no)

HR 2.18 (95\% Cl 1.55-3.08)

OR 1.8 (95\% Cl 1.3-2.6)
1600

100

Range 35-83

BDI/CES-D

No details

Home PSG

5 years

Not reported (small given wide confidence interval)

Logistic regression (?) Model: no details

Severe OSA $(A H I \geqslant 30)$

OR 2.9 (95\% Cl 1.19-6.92)

DD: depressive disorder; ICD: International Classification of Diseases; BDI: Beck depression inventory; CES-D: Centre for Epidemiologic Studies depression scale; PSG: polysomnography; AHI: apnoea-hypopnoea index; GEEM: generalised estimating equation model; BMI: body mass index; CVD: cardiovascular disease; CHD: coronary heart disease; OR: odds ratio; HR: hazard ratio.

administrative data housed at the Institute for Clinical Evaluative Sciences (ICES, Toronto, ON, Canada) from 1991 to 2015. Details regarding the cohort are provided elsewhere [14].

\section{Data sources}

Clinical data

The clinical database includes demographic, clinical and polysomnographic (PSG) information that has been collected consistently at our sleep laboratory for research purposes. Details regarding the variables collected are reported elsewhere and provided in online supplementary table E1 [14].

\section{Provincial health administrative data}

Residents of Ontario have universal public health insurance that covers all medically necessary services. Since 1991, ICES has housed high-quality administrative datasets on publicly funded services provided, including individual-level information on physician claims, hospitalisation and emergency department visits within Ontario [15]. The accuracy of these datasets has been validated previously [16, 17]. For all insured Ontario residents who have been diagnosed with OSA by a sleep physician, funding is provided for continuous, autotitrating and bilevel positive airway pressure (PAP) systems and this has been documented in the Assistive Devices Programme database from 2000 onwards [18]. The Ontario Mental Health Reporting System captures clinical, administrative and resource information on all adult inpatient mental health beds in Ontario since October 2005 [19]. The data are constantly evaluated, documented and improved according to Canadian Institute for Health Information's data quality framework [20].

These datasets were linked using unique encoded identifiers. A description of the ICES datasets is available at https://datadictionary.ices.on.ca/Applications/DataDictionary/Default.aspx. The full dataset creation plan is available from the authors upon request.

\section{Study participants}

A consecutive sample of all adults referred with suspected OSA who underwent a first diagnostic level 1 PSG at a large academic hospital between 1994 and 2010 was included. Based on the total apnoea- 
hypopnoea index (AHI), individuals were classified as no OSA (AHI $<5$ events $\cdot \mathrm{h}^{-1}$ ) or mild (AHI 5-14 events $\cdot h^{-1}$ ), moderate (AHI 15-30 events. $h^{-1}$ ) or severe OSA (AHI $>30$ events $\cdot h^{-1}$ ).

\section{Exposure: presence of OSA and its severity}

Objective and subjective measures of OSA severity were considered. Objective measures of OSA severity examined were AHI, sleep time spent with arterial oxygen saturation $\left(\mathrm{SaO}_{2}\right)<90 \%$, arousal index and number of awakenings in total sleep time. Self-reported symptoms of OSA taken into consideration were presence of self-reported restless sleep, waking unrefreshed, morning headaches, memory and concentration impact (trouble concentrating or remembering things), witnessed apnoea, observed snoring and daytime sleepiness. Daytime sleepiness was measured using the Epworth sleepiness scale [21].

Sleep disruption, insomnia and mismanagement of sleep associated with OSA may alter state of mind, potentially causing depression; conversely, depression is often associated with disturbed sleep and insomnia without evidence of OSA $[22,23]$. Thus, total sleep time measured using PSG and hypnotic use (a positive response to the question: "Do you ever take medication to help you fall asleep?") were considered as measures of sleep quality.

\section{Outcomes: depression}

The primary outcome was the time from the diagnostic sleep study to a subsequent hospitalisation resulting primarily from diagnosis of depression (table 2 and online supplementary table E2). Hospitalisation was chosen as a well-defined, validated and standardised measure that is of interest to patients, doctors and policy makers, to minimise misdiagnosis between OSA and depression resulting from shared symptomology, and due to the unknown exact time of the incident depression. Chart re-abstraction studies have shown that the most responsible diagnosis is coded accurately ( $\kappa 0.80,95 \%$ CI $0.74-0.87$ for International Classification of Diseases-10 code F32: depressive episode) [16].

The secondary outcome, prevalent physician-diagnosed depression, was defined as any inpatient or outpatient visit with a diagnosis of depression at any time prior to the sleep study (table 2). We made our definition of prior depression as all-encompassing as possible to maximise sensitivity. Although there is no validated health administrative data algorithm to define physician-diagnosed prevalent depression, the accuracy of administrative data for identifying mental health service provision in primary care compared with clinical data in Toronto (ON, Canada) has been shown to be $86.8 \%$ [24].

Finally, we identified separately a subgroup of individuals hospitalised for major depression only.

\section{Potential confounders and risk factors considered in the statistical model}

The following variables, which are known to be associated with depression or were included in statistical models in similar studies $[10,11,25]$, were considered from the clinical data: demographics (age, sex and body mass index (BMI)), self-reported smoking (current, ex- or never-smoker) and employment (yes/no) status and alcohol intake (consuming more than seven alcoholic beverages per week). From health

TABLE 2 Definition of depression from health administrative data used in the study

\section{Diseases}

Hospitalisation for depression (from CIHI/DAD and Ontario mental health reporting system)
Outpatient visits for depression (from OHIP dataset)

\section{Codes}

ICD-9-CM (prior to fiscal year 2002):

296.2, 296.3, 296.5, 300.4, 309.x and 311

Major depression subgroup: 296.2 and 296.3

ICD-10-CA (after fiscal year 2002):

F20.4, F31.3-F31.5, F32.x, F33.x, F34.1, $F 41.2$ and $F 43.2$

Major depression subgroup: F32.x and F33.x DSM-IV codes:

29620-29626, 29630-29636, 29650-29656, 30040, 30900,30928 and 31100

Major depression subgroup: 2962 and 2963 OHIP codes:

311 (depression) and 300 (group of anxiety or depression-related illnesses)

CIHI: Canadian Institute for Health Information; DAD: Discharge Abstract Database (inpatient hospitalisations); OHIP: Ontario Health Insurance Plan database ICD: International Classification of Diseases, CM: clinical modification; CA: Canada; DSM-IV: Diagnostic and Statistical Manual of Mental Disorders, 4th edition. 
administrative data we assessed baseline comorbidities (prior hypertension, diabetes, cardiovascular diseases, chronic obstructive pulmonary disease, dementia and cancer), the presence of alcoholism (physician-diagnosed alcohol intoxication and dependence), rural residence, income (via patient postal code) and prior primary care utilisation (number of patient's primary care visits within the year prior to baseline). Details on definitions are provided in the online supplementary table E3.

\section{Analyses}

Descriptive statistics were used to characterise our population of interest.

\section{Primary analyses}

Cox regression was used to investigate an association between OSA symptoms and severity at baseline and the incidence of hospitalisation for depression. Results were expressed as hazard ratios (HRs) and 95\% confidence intervals. Restricted cubic spline transformations were applied for continuous explanatory variables if nonlinearity was observed [26]. The resulting standardised HRs compared the 75th and 25th percentiles, allowing comparison of the HRs on a common scale [26]. Anticipating that a relatively small number of hospitalisations would occur during follow-up, and thus the number of variables that could be included in the multivariable model would be small, we decided a priori to test the effect of each exposure variable individually, controlling for confounders and risk factors selected based on the literature review, expert opinion and backward step-down variable deletion [27]. Because self-reported information concerning alcohol intake was unavailable in $>20 \%$ of the study patients, for the primary analyses we considered only alcoholism defined from health administrative data.

Although we had planned to investigate the association between OSA and hospitalised depression both in individuals with and without prior depression, the vast majority of hospitalisations (97\%) occurred in patients with prior depression. Thus, for our primary analyses, we focused on the population with prior depression at baseline.

\section{Secondary analyses}

Logistic regression was used to investigate the association between baseline OSA symptoms and severity and prevalent depression. All exposure variables and predefined potential confounders and risk factors were considered in the statistical models. In addition, interactions between AHI or $\mathrm{SaO}_{2}$ and level of daytime sleepiness, BMI, age and sex were considered. Finally, in the post-2000 cohort with information on PAP treatment initiation, the final model was refitted with the addition of a variable on treatment initiation (yes/no).

Missing data on the variables considered, except alcohol intake, ranged from $0.69 \%$ (AHI) to $10.1 \%$ (time spent with $\mathrm{SaO}_{2}<90 \%$ ). To impute missing values, we used a multivariate imputation by chained equations approach to generate five complete datasets [28]. Given that our results were confirmed on imputed datasets, for a unified presentation the findings presented are based on the original dataset (completed case analyses).

All statistical analyses were performed in the secure environment of ICES following provincial privacy standards using $\mathrm{R}$ version 2.15.2. The ethics committees of all institutions involved (St Michael's Hospital and ICES) approved the study.

\section{Results}

Mean AHI was 25 events $\mathrm{h}^{-1}$ in the 10149 subjects included in our analyses (mean age 50 years, 62\% male); $21 \%$ had AHI $<5$ events. $\mathrm{h}^{-1}$ and $29 \%$ had AHI $>30$ events. $\mathrm{h}^{-1}$. Among individuals with suspected OSA, 7536 (74.3\%) had been diagnosed with depression prior to their diagnostic sleep study. The majority of depression diagnoses were made in a primary care setting, with only 211 (2.8\%) subjects hospitalised for major depression prior to their sleep study. Those with baseline depression were more likely to be female, current smokers, to abuse alcohol, to have lower income, unemployed and have more comorbidities. Participants with depression had more self-reported symptoms of OSA, but less severe OSA as measured by AHI (table 3).

\section{Relationships between OSA symptoms and severity and baseline depression}

Controlling for sex, age, BMI, smoking, alcohol consumption, comorbidity, income and employment, symptoms of OSA such as waking unrefreshed and reporting trouble concentrating or remembering things and use of hypnotics, but not objective measures of its severity $(p>0.25)$ were significantly associated with baseline depression (table 4). Other factors significantly associated with prior depression were female sex, more severe comorbidities and alcoholism, being a current smoker and more frequent exposure to primary care. The effect of OSA symptoms became nonsignificant when the statistical model was refitted for the prior hospitalisation for major depression as an outcome (not shown). 
TABLE 3 Characteristics of subjects with suspected obstructive sleep apnoea (OSA) by presence of baseline depression and by incident hospitalisation for depression

\begin{tabular}{|c|c|c|c|c|}
\hline & \multirow[t]{2}{*}{ Without prior depression } & \multirow[t]{2}{*}{ With prior depression } & \multicolumn{2}{|c|}{ With prior depression } \\
\hline & & & Nonhospitalised & $\begin{array}{c}\text { Hospitalised } \\
\text { during follow-up }\end{array}$ \\
\hline Male & 2019 (77.3) & $4265(56.6)$ & 4208 (57) & $57(43)$ \\
\hline Age years & $48(37-59)$ & $49(40-59)$ & $49(40-59)$ & $45.0(38.0-54.0)$ \\
\hline \multicolumn{5}{|l|}{ Smoking status ${ }^{\#}$} \\
\hline Current & $393(16)$ & $1446(21)$ & $1402(21)$ & $44(36)$ \\
\hline Ex-smoker & $475(20)$ & $1394(20)$ & $1381(20)$ & $13(11)$ \\
\hline Never-smoker & $1521(63)$ & 4085 (58) & 4021 (59) & $64(53)$ \\
\hline Alcoholism & $52(2.0)$ & $564(7.5)$ & $530(7.2)$ & $34(25.8)$ \\
\hline Hypnotic use $\#$ & $392(16)$ & $2413(34)$ & 2334 (33) & $79(64)$ \\
\hline \multicolumn{5}{|l|}{ Income status ${ }^{\#}$ quintiles } \\
\hline 5 & 853 (33) & $2012(27)$ & 1977 (27) & $35(27)$ \\
\hline Employed $^{\#}$ & $1761(72)$ & 4399 (62) & $4348(63)$ & $51(42)$ \\
\hline \multicolumn{5}{|l|}{ Presence of prior comorbidities at baseline } \\
\hline Hypertension & $876(33.5)$ & $2716(36.0)$ & $2672(36.1)$ & 44 (33.3) \\
\hline Myocardial infarction & $93(3.6)$ & $307(4.1)$ & $300(4.1)$ & 7 (5.3) \\
\hline $\mathrm{CHF}$ & 179 (6.9) & $447(5.9)$ & $437(5.9)$ & $10(7.6)$ \\
\hline Diabetes & $344(13.2)$ & $1130(15.0)$ & $1113(15.0)$ & $17(12.9)$ \\
\hline COPD & $241(9.2)$ & $1017(13.5)$ & $998(13.5)$ & $19(14.4)$ \\
\hline Asthma & $366(14.0)$ & $1465(19.4)$ & $1373(18.5)$ & $36(27.3)$ \\
\hline \multicolumn{5}{|l|}{ ADG categories } \\
\hline Low & $1236(47)$ & $1635(22)$ & $1617(22)$ & $18(14)$ \\
\hline Moderate & $953(42)$ & $3167(42)$ & $3122(42)$ & $45(34)$ \\
\hline Memory impacted & $1063(44)$ & $4353(62)$ & $4267(62)$ & $86(72)$ \\
\hline Daytime sleepiness & 906 (37) & 2751 (39) & 2704 (39) & 47 (39) \\
\hline ESS score (total 24) & $8(5-11)$ & $8(5-12)$ & $8(5-12)$ & $9(4-12)$ \\
\hline \multicolumn{5}{|l|}{ PSG indexes } \\
\hline Sleep efficiency \% & $82.4(70.4-89.8)$ & $81.2(69.2-88.9)$ & $81.2(69.3-88.9)$ & $80.7(63.0-88.3)$ \\
\hline Total sleep time $\mathrm{h}$ & $5.8(4.9-6.4)$ & $5.7(4.9-6.4)$ & $5.74(4.87-6.42)$ & $5.7(4.6-6.3)$ \\
\hline $\mathrm{AHI}$ total events $\cdot \mathrm{h}^{-1}$ & $18.7(7.3-40.2)$ & $15.0(5.7-32.9)$ & $15.1(5.7-33.0)$ & $10.9(3.7-28.9)$ \\
\hline Mean $\mathrm{SaO}_{2} \%$ & $94.8(93.4-96.0)$ & $94.9(93.5-96.1)$ & $94.9(93.5-96.1)$ & $95.2(93.5-96.4)$ \\
\hline Sleep time spent with $\mathrm{SaO}_{2}<90 \% \mathrm{~min}$ & $0.9(0.0-13.2)$ & $0.4(0.0-7.8)$ & $0.4(0.0-7.9)$ & $0.1(0.0-5.7)$ \\
\hline Initiated PAP treatment & $1046(40.0)$ & $2988(39.7)$ & $2935(39.6)$ & $52(39.4)$ \\
\hline
\end{tabular}

Data are presented as $\mathrm{n}(\%)$ or median (interquartile range), unless otherwise stated. BMI: body mass index; CHF: chronic heart failure; COPD: chronic obstructive pulmonary disease; ADG: Johns Hopkins aggregated diagnosis groups; ESS: Epworth sleepiness scale; PSG: polysomnography; $\mathrm{AHI}$ : apnoea-hypopnoea index; $\mathrm{SaO}_{2}$ : arterial oxygen saturation; PAP: positive airway pressure. \# : numbers may not add to total because of missing values.

Relationship between OSA symptoms and severity with incidence of hospitalisation for depression Over a median follow-up of 9.7 years, 136 (1.3\%) participants were hospitalised for depression; among them, 103 (75.7\%) were hospitalised for major depression, giving an incident rate of 1.37 per 1000 person.year ${ }^{-1}$. The majority (97.1\%) of hospitalisations for depression occurred in subjects with baseline depression. Therefore, subsequent analyses focused on this population only. 
TABLE 4 The relationship between individual baseline characteristics and baseline physician-diagnosed and hospitalised depression in multivariable logistic regression analyses

Prior depression, any diagnosis

$1.43(1.26-1.62)$

$1.54(1.36-1.72)$

$1.83(1.61-2.07)$

$1.03(0.94-1.13)$

$0.97(0.90-1.03)$

$1.88(1.64-2.16)$

$1.26(1.08-1.48)$

$2.19(1.84-2.60)$

$2.83(2.02-3.95)$

$1.66(1.52-1.82)$

$1.27(1.10-1.47)$

$1.12(0.97-1.29)$

$1.07(0.91-1.26)$

$0.89(0.78-1.02)$
Prior hospitalisation

for major depression

$1.40(0.84-2.32)$

$1.16(0.80-1.70)$

$2.05(1.46-2.89)$

$0.67(0.52-0.86)$

$1.18(1.01-1.37)$

$2.90(2.07-4.05)$

$1.41(0.98-2.04)$

$1.46(0.87-2.44)$

$4.29(2.92-6.30)$

$1.12(1.03-1.22)$

$2.14(1.49-3.07)$

$0.86(0.52-1.42)$

$1.39(0.88-2.21)$

$2.38(1.67-3.40)$

Employment status: no versus yes

Data are presented as OR (95\% CI). OSA: obstructive sleep apnoea; BMI: body mass index; ADG: Johns Hopkins aggregated diagnosis groups.

Among individuals with baseline depression, those subsequently hospitalised were more likely to be female, younger, current smokers, obese, unemployed, to abuse alcohol, have a lower income and more comorbidities, take hypnotics, to report symptoms of OSA more often, and had less severe OSA as measured by AHI (table 3). In individuals with severe OSA and prior depression, PAP treatment had been initiated in $75 \%$ of those subsequently hospitalised for depression and $74 \%$ of those who were not.

A significant crude effect of OSA symptoms (waking unrefreshed and impact on memory and concentration) on hospitalised depression became nonsignificant after controlling for confounders (table 5). An increase in AHI was not significantly associated with the outcome: adjusted hazard ratio (33 versus 6 events $\cdot \mathrm{h}^{-1}$ ) of 1.13 (95\% CI $\left.0.91-1.40\right)$. Factors associated with incident hospitalised depression were: female sex, younger age, alcoholism, use of hypnotics and unemployment (figure 1). Results did not change substantially after additionally controlling for initiation of PAP treatment (table 5).

The results were confirmed on the entire sample (including individuals without prior depression at baseline) and on five imputed datasets (not shown). No significant interactions were found.

\section{Discussion}

In a large clinical cohort with suspected OSA who underwent a diagnostic sleep study, physician-diagnosed depression was highly prevalent. Subjects with depression at baseline were more likely to report symptoms suggesting OSA, such as waking unrefreshed and experiencing trouble concentrating or remembering things, but did not differ from those without depression with respect to objective measures of OSA severity. The hospitalisation rate for depression observed in our population was similar to the hospitalisation rate for depression in the sex- and age-standardised general population in Ontario and in Canada [29-31]. Furthermore, neither OSA symptoms nor objective measures of OSA severity, as measured by polysomnography, were independent predictors of hospitalisation for depression during the follow-up period of almost one decade.

Our findings suggest that previously reported associations between OSA and depression probably reflect diagnostic misclassification based on overlapping diagnostic criteria. For example, both the Beck Depression Inventory [32] and the Diagnostic and Statistical Manual of Mental Disorders (DSM)-IV criteria for diagnosis of depression include no fewer than five symptoms that could easily reflect OSA: loss of energy, changes in sleeping pattern, irritability, difficulty with concentration and fatigue. To our knowledge, this is the first study to examine the longitudinal relationship between OSA, using both subjective and objective measures of severity, and severe depression resulting in hospitalisation, a robust 
TABLE 5 The relationship between obstructive sleep apnoea (OSA) and sleep-related factors at baseline and incident hospitalisation for depression in Cox regression analyses

\begin{tabular}{|c|c|c|c|c|c|}
\hline \multicolumn{2}{|c|}{ Univariable } & \multicolumn{2}{|c|}{ Multivariable } & \multicolumn{2}{|c|}{$\begin{array}{l}\text { Additional adjustment } \\
\text { for treatment initiation }\end{array}$} \\
\hline $\begin{array}{c}\text { Hazard ratio } \\
(95 \% \mathrm{Cl})\end{array}$ & $\mathrm{p}$-value & $\begin{array}{c}\text { Hazard ratio } \\
(95 \% \mathrm{CI})\end{array}$ & p-value & $\begin{array}{c}\text { Hazard ratio } \\
\text { (95\% CI) }\end{array}$ & p-value \\
\hline
\end{tabular}

\section{Presence of self-reported symptoms of OSA}

Restless sleep: yes versus no

Morning headaches: yes versus no

Wake unrefreshed: yes versus no

Stop breathing: yes versus no

Snoring: yes versus no

Memory and concentration impact: yes versus no

ESS/24: 12 versus 5

$1.41(0.99-2.03)$
$1.40(0.98-2.00)$
$\mathbf{2 . 3 2}(1.31-4.13)$
$0.96(0.66-1.40)$
$1.18(0.72-1.92)$
$\mathbf{1 . 5 1}(1.02-2.25)$
$1.02(0.79-1.30)$

$0.06 \quad 1.10(0.74-1.62)$

0.07

0.64

0.76

$1.09(0.74-1.61)$

0.66

$<0.01$

$1.06(0.72-1.56)$

0.27

$1.04(0.71-1.53)$

$1.38(0.74-2.55)$

0.82

$0.83 \quad 1.01(0.67-1.53) \quad 0.96$

$0.52 \quad 1.42(0.82-2.44) \quad 0.21$

$0.95(0.62-1.45)$

0.31

$1.33(0.77-2.31)$

0.80

$\begin{array}{lllll}\mathbf{0 . 0 4} & 0.86(0.56-1.31) & 0.48 & 0.85(0.56-1.31) & 0.47\end{array}$

Polysomnographic variables

TST: 4.9 versus $6.4 \mathrm{~h}^{-1}$

Total AHI: 33 versus 6 events. $h^{-1}$

$1.23(1.01-1.49)$

$0.96(0.77-1.18)$

$0.90-1.09(0.84-1.41)$

0.57

$1.07(0.83-1.39)$

0.60

Sleep time spent with $\mathrm{SaO}_{2}<90 \%$ : 8 versus 0 min

Arousal index: 38 versus 14 events. $h^{-1}$

$1.02(0.99-1.05)$

$\mathbf{0 . 0 4} 1.13(0.91-1.40)$

0.25

0.68

$1.13(0.91-1.40)$

0.27

$1.14(0.92-1.41)$

$1.05(0.81-1.37)$

0.22

$0.88(0.70-1.10)$

0.22

$1.02(0.99-1.05)$

0.13

$1.02(0.99-1.05)$

0.69

$0.25 \quad 1.00(0.80-1.26)$

0.99

$0.91(0.70-1.18)$

0.23

$0.87(0.71-1.07)$

0.20

$0.98(0.79-1.20)$

0.81

$0.96(0.77-1.18)$

0.47

0.67

\section{Medical history}

Hypnotic use ${ }^{\text {n }}$ yes versus no

$3.64(2.52-5.27)$

$2.79(1.89-4.12)$

$<0.0001$

$2.84(1.92-4.20)$

$<0.0001$

Bold type represents statistical significance. ESS: Epworth sleepiness scale; TST: total sleep time; AHI: apnoea-hypopnoea index; SaO ${ }_{2}$ arterial oxygen saturation. "\#: the effect of OSA-related variables tested separately in the statistical model controlling for sex, age, smoking and employment status, hypnotic use and alcoholism (variables selected by backward step-down variable deletion procedure); ": controlling for sex, age, smoking and employment status and alcoholism.

end-point that is unlikely to result from diagnostic confusion due to overlapping symptoms between OSA and depression.

However, our findings cannot exclude a potential link between OSA and milder forms of depression. It is conceivable that OSA predisposes to milder cases of depression, but is not a risk factor for severe, refractory depression that often leads to hospitalisation. That different depression phenotypes may have different underlying risk factors is a possibility that cannot be excluded by our study. However, other recognised risk factors for depression including sex, smoking, employment status and alcohol abuse were found to be significantly associated with incident hospitalised depression in our study, suggesting that the risk factors for mild and severe depression are similar. Furthermore, since a pre-existing diagnosis of depression has been found to be the strongest risk factor for subsequent hospitalisation for depression, it seems likely that if objective severity of OSA were truly biologically linked to milder depression, it should also predispose to more severe depression, which was not found in our study.

FIGURE 1 Results from multivariable Cox regression model presented as hazard ratios $195 \%$ CI). AHI: apnoea-hypopnoea index.

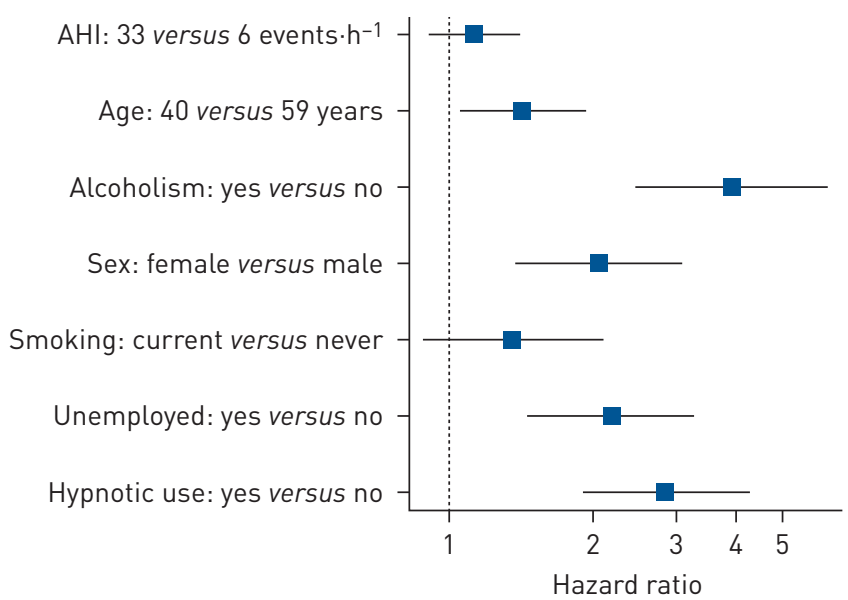


Consistent with other studies, we found a high prevalence of depression in patients with suspected OSA compared to the community in general [1]. The high prevalence of previously diagnosed depression in our cohort may be explained by two reasons. Firstly, there is a high potential for OSA to be misdiagnosed as depression given a significant overlap in symptoms [4, 22, 33, 34]. Existing evidence regarding the accuracy and case yield of depression screening tools in the primary care setting suggested that $>80 \%$ of positive screening results are probably false positives (indicating presence of depression, when in reality it is not) [35]. Secondly, there likely to be a referral bias: patients with depression are more likely to be referred for sleep studies because of symptoms suggesting OSA, even when OSA is not present.

Strongly supporting this notion was our finding that symptoms that are related to OSA, but not objective measures of OSA, were significantly associated with prior depression. In fact, patients with prior depression were actually less likely to have OSA than those without depression, demonstrating the referral bias. While referral bias is a limitation of our study due to its clinic-based design, it has significant advantages compared to community-based studies. Community-based studies $[36,37]$ have relatively few cases of severe OSA, whereas $29 \%$ of our cohort were severe cases. In fact, the number of severe OSA cases in our cohort is an order of magnitude greater than in the most prominent community-based cohorts, thereby increasing our ability to detect any adverse effects.

Furthermore, where OSA is present, its effective treatment often results in improvement of OSA-related depressive symptoms $[7,8]$. The findings that symptoms suggestive of depression occur commonly in association with OSA and are responsive to CPAP therapy indicate a need for systematic screening of depressed populations for OSA, especially among individuals with depression who fail to respond to standard antidepressant treatment.

In our study, we found hypnotic use to be significantly associated with both prevalent and incident depression. This was not surprising, since others have indicated an association between hypnotic use, sleep-related breathing disorder and depression $[4,38]$. Furthermore, alcohol and hypnotic medication use may decrease upper airway muscle tone precipitation or exacerbating OSA [39].

Compared to prior studies published, we have the largest sample, longest follow-up time, a comprehensive set of polysomnographic variables collected in a sleep laboratory and a robust end-point: hospitalisation for depression (table 1) [10-12]. In contrast, any study purporting to show an association between OSA and depression based on questionnaires or physician diagnosis in a primary care setting must contend with the strong possibility of diagnostic confusion due to overlapping symptoms.

Our findings are limited by the observational single-centre study design, which may affect the generalisability of our results and increase the potential for unmeasured confounding. For example, we did not have information on level of education, treatment for depression and adherence with antidepressant or PAP treatment over time. One could hypothesise that severe OSA did not increase the risk of hospitalisation for depression because most of these patients were successfully treated using CPAP. However, the proportion of patients who initiated CPAP treatment did not differ significantly between those with severe OSA who were hospitalised for depression compared to those who were not. Furthermore, it is well known that CPAP adherence rates are generally low [40,41], making it unlikely that treatment effects were enough to extinguish a true relationship in such a large cohort.

Furthermore, the administrative diagnostic code used to define baseline depression is among the most frequently used diagnostic codes in primary care in Ontario and includes a number of mental health disorders other than depression. However, we intended to make our definition as broad as possible in order not to miss any cases of prevalent depression. In contrast, the Ontario administrative code for hospitalised depression, our primary end-point, is specific to that disorder. Importantly, the effects of sex, smoking, income, employment status and alcohol abuse on incident depression in our study are similar to other studies, validating the clinical significance of our primary outcome [29]. Assessment of the use of hypnotics may be limited by recall bias. However, the distribution and effect of hypnotic use in our cohort were similar to that reported in other studies $[4,38]$. Finally, although we did not find a statistically significant association between objective OSA severity and hospitalisation for depression, we may have insufficient power due to the relatively small number of events.

\section{Conclusions}

In a large clinical cohort with suspected OSA, physician-diagnosed depression was highly prevalent. Subjects with depression at baseline were more likely to report symptoms suggesting OSA, but did not differ from those without depression with respect to objective measures of OSA severity. Furthermore, neither OSA symptoms nor objective measures of OSA severity were independent predictors of hospitalisation for depression during the follow-up period of almost one decade. Our findings suggest that previously reported associations between OSA and depression may be explained by links between OSA and milder depression, or reflect diagnostic misclassification based on overlapping diagnostic criteria. 


\section{Acknowledgements}

Parts of this material are based on data and information compiled and provided by the Canadian Institute for Health Information (CIHI). However, the analyses, conclusions, opinions and statements expressed herein are those of the author, and not necessarily those of CIHI. Parts of this material are based on data and information provided by Cancer Care Ontario (CCO). The opinions, results, view, and conclusions reported in this paper are those of the authors and do not necessarily reflect those of CCO. No endorsement by CCO is intended or should be inferred.

The authors thank Victor Hoffstein for creating and maintaining the St Michael's Hospital (Toronto, ON, Canada) sleep study database.

Author contributions: all co-authors were involved in the following: study design, ethics board application, interpretation of data, revising the manuscript critically for the accuracy and important intellectual content and final approval of the version to be published. T. Kendzerska additionally was involved in the following: literature search, study conception, obtaining administrative data, analyses of data and drafting of the manuscript. R.S. Leung additionally was involved in study conception and drafting of the manuscript, and is a custodian of the sleep database housed at St Michael's Hospital, from which the study sample was extracted. A.S. Gershon additionally was involved in obtaining administrative data and data analyses. G.A. Tomlinson additionally was involved in data analyses. All authors had full access to all statistical reports and tables in the study and can take responsibility for the integrity of the data and the accuracy of the data analysis. Only T. Kendzerska had full access to all the data including raw data. The lead author, R.S. Leung, affirms that the manuscript is an honest, accurate, and transparent account of the study being reported; that no important aspects of the study have been omitted; and that any discrepancies from the study as planned have been explained.

\section{References}

1 BaHammam AS, Kendzerska T, Gupta R, et al. Comorbid depression in obstructive sleep apnea: an under-recognized association. Sleep Breath 2016; 20: 447-456.

2 Sharafkhaneh A, Richardson P, Hirshkowitz M. Sleep apnea in a high risk population: a study of Veterans Health Administration beneficiaries. Sleep Med 2004; 5: 345-350.

3 Ejaz SM, Khawaja IS, Bhatia S, et al. Obstructive sleep apnea and depression: a review. Innov Clin Neurosci 2011; 8: 17-25.

4 Björnsdóttir E, Benediktsdóttir B, Pack AI, et al. The prevalence of depression among untreated obstructive sleep apnea patients using a standardized psychiatric interview. J Clin Sleep Med 2016; 12: 105-112.

5 Macey PM, Woo MA, Kumar R, et al. Relationship between obstructive sleep apnea severity and sleep, depression and anxiety symptoms in newly-diagnosed patients. PLoS One 2010; 5: e10211.

6 Gupta MA, Simpson FC. Obstructive sleep apnea and psychiatric disorders: a systematic review. J Clin Sleep Med 2015; 11: 165-175

7 Edwards C, Mukherjee S, Simpson L, et al. Depressive symptoms before and after treatment of obstructive sleep apnea in men and women. J Clin Sleep Med 2015; 11: 1029-1038.

8 Povitz M, Bolo CE, Heitman SJ, et al. Effect of treatment of obstructive sleep apnea on depressive symptoms: systematic review and meta-analysis. PLoS Med 2014; 11: e1001762.

9 Gagnadoux F, Le Vaillant M, Goupil F, et al. Depressive symptoms before and after long-term CPAP therapy in patients with sleep apnea. Chest 2014; 145: 1025-1031.

10 Peppard PE, Szklo-Coxe M, Hla KM, et al. Longitudinal association of sleep-related breathing disorder and depression. Arch Intern Med 2006; 166: 1709-1715.

11 Chen $\mathrm{YH}$, Keller JK, Kang JH, et al. Obstructive sleep apnea and the subsequent risk of depressive disorder: a population-based follow-up study. J Clin Sleep Med 2013; 9: 417-423.

12 Lang C, Appleton SL, Vakulin A, et al. Obstructive sleep apnea (OSA) and excessive daytime sleepiness (EDS) are independently associated with depression in a community based population of Australian men. Am J Respir Crit Care Med 2015; 191: A3934.

13 Kendzerska T, Mollayeva T, Gershon AS, et al. Untreated obstructive sleep apnea and the risk for serious long-term adverse outcomes: a systematic review. Sleep Med Rev 2014; 18: 49-59.

14 Kendzerska T, Gershon AS, Hawker G, et al. Obstructive sleep apnea and risk of cardiovascular events and all-cause mortality: a decade-long historical cohort study. PLoS Med 2014; 11: e1001599.

15 Institute for Clinical Evaluative Sciences (ICES). Improving Health Care Data in Ontario. ICES Investigative Report. Toronto, ICES, 2005.

16 Juurlink D, Preyra C, Croxford R, et al. Canadian Institute for Health Information Discharge Abstract Database: a Validation Study. Toronto, Institute for Clinical Evaluative Sciences, 2006.

17 Goel V, Williams JI, Anderson GM, et al., eds. Patterns of Health Care in Ontario. 2nd Edn. Ottawa, Canadian Medical Association [for] the Institute for Clinical Evaluative Sciences in Ontario, 1996.

18 Assistive Devices Program and Ministry of Health and Long-term Care. Respiratory Policy and Administration Manual. May 2016. www.health.gov.on.ca/en/pro/programs/adp/policies_procedures_manuals/docs/respiratory_ equipment_manual.pdf Date last accessed: March 26, 2017.

19 Canadian Institute for Health Information. Ontario Mental Health Reporting System Metadata. 2013-2014. www.cihi.ca/en/types-of-care/specialized-services/mental-health-and-addictions/ontario-mental-health-reporting Date last accessed: April 12, 2015.

20 Canadian Institute for Health Information. Ontario Mental Health Reporting System, Data Quality Documentation, 2013-2014. Ottawa, Canadian Institute for Health Information, 2014.

21 Johns MW. A new method for measuring daytime sleepiness: the Epworth sleepiness scale. Sleep 1991; 14: $540-545$.

22 Lee W, Lee SA, Chung YS, et al. The relation between apnea and depressive symptoms in men with severe obstructive sleep apnea: mediational effects of sleep quality. Lung 2015; 193: 261-267.

23 Horner RL. The Universal Pastime: Sleep and Rest Explained. Pennsauken, BookBaby, 2014.

24 Steele LS, Glazier RH, Lin E, et al. Using administrative data to measure ambulatory mental health service provision in primary care. Med Care 2004; 42: 960-965. 
25 Hölzel L, Härter M, Reese C, et al. Risk factors for chronic depression - a systematic review. J Affect Disord 2011; 129: 1-13.

26 Harrell FE. Regression Modeling Strategies: With Applications to Linear Models, Logistic Regression, and Survival Analysis. New York, Springer, 2001

27 Atkinson AC. A note on the generalized information criterion for choice of a model. Biometrika 1980; 67: 413-418.

28 Azur MJ, Stuart EA, Frangakis C, et al. Multiple imputation by chained equations: what is it and how does it work? Int J Methods Psychiatr Res 2011; 20: 40-49.

29 Bryan S. A Report on Mental Illnesses in Canada. Ottawa, Health Canada, 2002.

30 Kasman N, Ardal S. The Geographic Variation of Depression and Health Service Utilization in Ontario. Toronto, Central East Health Information Partnership, 2005.

31 Brien S, Grenier L, Kapral M, et al. Taking Stock: a Report on the Quality of Mental Health and Addictions Services in Ontario. Toronto, Health Quality Ontario and the Institute for Clinical Evaluative Sciences, 2015.

32 Beck AT, Steer RA, Garbin MG. Psychometric properties of the Beck Depression Inventory: twenty-five years of evaluation. Clin Psychol Rev 1988; 8: 77-100.

33 Ishman SL, Cavey RM, Mettel TL, et al. Depression, sleepiness, and disease severity in patients with obstructive sleep apnea. Laryngoscope 2010; 120: 2331-2335.

34 Luik AI, Noteboom J, Zurbier LA, et al. Sleep apnea severity and depressive symptoms in a population-based study. Sleep Health 2015; 1: 128-132.

35 Thombs BD, Arthurs E, El-Baalbaki G, et al. Risk of bias from inclusion of patients who already have diagnosis of or are undergoing treatment for depression in diagnostic accuracy studies of screening tools for depression: systematic review. BMJ 2011; 343: d4825.

36 Young T, Finn L, Peppard PE, et al. Sleep disordered breathing and mortality: eighteen-year follow-up of the Wisconsin sleep cohort. Sleep 2008; 31: 1071-1078.

37 Redline S, Yenokyan G, Gottlieb DJ, et al. Obstructive sleep apnea-hypopnea and incident stroke: the sleep heart health study. Am J Respir Crit Care Med 2010; 182: 269-277.

38 Li CT, Bai YM, Lee YC, et al. High dosage of hypnotics predicts subsequent sleep-related breathing disorders and is associated with worse outcomes for depression. Sleep 2014; 37: 803-809.

39 Heck T, Zolezzi M. Obstructive sleep apnea: management considerations in psychiatric patients. Neuropsychiatr Dis Treat 2015; 11: 2691-2698.

40 Rotenberg BW, Murariu D, Pang KP. Trends in CPAP adherence over twenty years of data collection: a flattened curve. J Otolaryngol Head Neck Surg 2016; 45: 43.

41 Sawyer AM, Gooneratne NS, Marcus CL, et al. A systematic review of CPAP adherence across age groups: clinical and empiric insights for developing CPAP adherence interventions. Sleep Med Rev 2011; 15: 343-356. 